\title{
Vibration of effects from diverse inclusion/ exclusion criteria and analytical choices: 9216 different ways to perform an indirect comparison meta-analysis
}

Clément Palpacuer ${ }^{1,2^{*}}$, Karima Hammas ${ }^{3,4}$, Renan Duprez ${ }^{5}$, Bruno Laviolle $e^{1,6,7}$, John P. A. Ioannidis ${ }^{8,9}$ and Florian Naudet ${ }^{1,6,7,8}$

\begin{abstract}
Background: Different methodological choices such as inclusion/exclusion criteria and analytical models can yield different results and inferences when meta-analyses are performed. We explored the range of such differences, using several methodological choices for indirect comparison meta-analyses to compare nalmefene and naltrexone in the reduction of alcohol consumption as a case study.

Methods: All double-blind randomized controlled trials (RCTs) comparing nalmefene to naltrexone or one of these compounds to a placebo in the treatment of alcohol dependence or alcohol use disorders were considered. Two reviewers searched for published and unpublished studies in MEDLINE (August 2017), the Cochrane Library, Embase, and ClinicalTrials.gov and contacted pharmaceutical companies, the European Medicines Agency, and the Food and Drug Administration. The indirect comparison meta-analyses were performed according to different inclusion/exclusion criteria (based on medical condition, abstinence of patients before inclusion, gender, somatic and psychiatric comorbidity, psychological support, treatment administered and dose, treatment duration, outcome reported, publication status, and risk of bias) and different analytical models (fixed and random effects). The primary outcome was the vibration of effects (VoE), i.e. the range of different results of the indirect comparison between nalmefene and naltrexone. The presence of a "Janus effect" was investigated, i.e. whether the 1st and 99th percentiles in the distribution of effect sizes were in opposite directions.
\end{abstract}

Results: Nine nalmefene and 51 naltrexone RCTs were included. No study provided a direct comparison between the drugs. We performed 9216 meta-analyses for the indirect comparison with a median of 16 RCTs (interquartile range $=12-21)$ included in each meta-analysis. The standardized effect size was negative at the 1st percentile $(-0.29$, favouring nalmefene) and positive at the 99 th percentile $(0.29$, favouring naltrexone). A total of $7.1 \%$ $(425 / 5961)$ of the meta-analyses with a negative effect size and $18.9 \%(616 / 3255)$ of those with a positive effect size were statistically significant $(p<0.05)$.

Conclusions: The choice of inclusion/exclusion criteria and analytical models for meta-analysis can result in entirely opposite results. VoE evaluations could be performed when overlapping meta-analyses on the same topic yield contradictory result.

\footnotetext{
* Correspondence: clement.palpacuer@gmail.com

${ }^{1}$ Centre d'Investigation Clinique INSERM 1414, Hôpital de Pontchaillou, 2 rue

Henri le Guilloux, 35033 Rennes cedex 9, France

${ }^{2}$ Department of Biostatistics, Institut de Cancérologie de I'Ouest Centre

René-Gauducheau, Saint-Herblain, France

Full list of author information is available at the end of the article
}

(c) The Author(s). 2019 Open Access This article is distributed under the terms of the Creative Commons Attribution 4.0 International License (http://creativecommons.org/licenses/by/4.0/), which permits unrestricted use, distribution, and reproduction in any medium, provided you give appropriate credit to the original author(s) and the source, provide a link to the Creative Commons license, and indicate if changes were made. The Creative Commons Public Domain Dedication waiver (http://creativecommons.org/publicdomain/zero/1.0/) applies to the data made available in this article, unless otherwise stated. 
(Continued from previous page)

Trial registration: This study was registered on October 19, 2016, in the Open Science Framework (OSF, protocol available at https://osf.io/7bq4y/).

Keywords: Meta-analysis, Vibration of effect, Alcoholism, Nalmefene, Naltrexone

\section{Background}

Meta-analyses have become very popular and widely used by public health decision-makers, pharmaceutical companies, and clinicians in their day-to-day practice. Conventional meta-analyses only consider direct evidence from randomized controlled trials. However, there is an increasing interest in obtaining evidence from indirect comparisons to fill the gaps in comparative effectiveness research. Both direct and indirect comparisons can be considered in large-scale network meta-analyses that rank multiple treatments [1]. However, the production of systematic reviews and meta-analyses has reached epidemic proportions [2-4], and sometimes, overlapping meta-analyses on the same topic obtain divergent results [5]. Discordant meta-analyses on the same topic have been a common recurring theme for diverse clinical questions [6-13]. The discordance is often due to the differences in the way that the meta-analyses were conducted, e.g. the studies considered to be eligible; how searches are performed; the models used for data synthesis; and how results are interpreted. For example, it has been shown that the estimation of treatment outcomes in meta-analyses differs depending on the analytic strategy used [14]. However, each topic and each case may have its own special considerations that explain the discordance. It would be useful to develop a heuristic approach that can systematically and objectively assess the potential for obtaining discordant results in any meta-analysis topic.

Here, we propose applying the vibration of effect (VoE) concept as a tool for examining the spectrum of results that can be obtained in meta-analyses when different choices are made. VoE describes the extent to which an effect may change under multiple distinct analyses, such as different model specifications in epidemiological research [15-17].

As a case study, we explored VoE in a very concrete and controversial example with regulatory implications: the comparison of nalmefene and naltrexone in the treatment of alcohol use disorders. Nalmefene is a 6-methyl derivative of naltrexone [18]. Both are opioid antagonists [19]. Naltrexone is an approved treatment, historically used in the post-withdrawal maintenance of alcohol abstinence, whereas nalmefene has only recently been approved by the European Medicine Agency (EMA) [20] for the indication of reducing alcohol consumption, based on a posteriori analyses of pivotal trials performed on subgroups of patients with a high risk of drinking [21]. Because nalmefene was the first drug approved in this new indication, the phase III clinical programme did not compare this compound with naltrexone or another active comparator. However, several health authorities have highlighted the need for comparative efficacy data between the two drugs [22]. The only available comparison of the two drugs is provided by two indirect and conflicting meta-analyses. The first meta-analysis, funded by Lundbeck (manufacturer of nalmefene), found an advantage of nalmefene over naltrexone [23] with subgroup analyses on nalmefene RCTs compared with naltrexone RCTs as a whole. This methodological choice probably resulted in a violation of the similarity assumption, which is necessary for indirect meta-analyses [21, 24]. The second meta-analysis, performed by our team, did not find any significant difference between nalmefene and naltrexone [25]. Thus, a single analytical choice (subgroup analysis of the data versus full analysis set) affected the estimated effect size for treatment effect. We hypothesized that many other methodological choices concerning the study selection process and statistical analyses can easily modify the effect sizes obtained and the inferences made from indirect comparisons. We thus explored VoE in a large number of indirect meta-analyses to compare nalmefene and naltrexone in the reduction of alcohol consumption, using different methodological choices concerning inclusion/exclusion criteria and analytical models.

\section{Methods \\ Design}

A standard protocol was developed and registered on October 19, 2016, before the beginning of the study, in the Open Science Framework (OSF, protocol available at https://osf.io/7bq4y/).

\section{Eligibility criteria}

All double-blind randomized controlled trials (RCTs) comparing nalmefene to naltrexone or one of these compounds to a placebo in the treatment of alcohol dependence (AD) or alcohol use disorders were included, regardless of other patient eligibility criteria, treatment modalities, or study duration. Study reports in English, French, German, Spanish, and Portuguese were considered. 


\section{Search strategy and study selection process}

Eligible studies were identified from PubMed/MEDLINE, the Cochrane Library, and Embase, including conference abstracts. Searches were initially conducted as part of a previous systematic review and meta-analysis that compared nalmefene, naltrexone, acamprosate, baclofen, and topiramate for the reduction of alcohol consumption [25]. The same algorithm was used for all electronic databases: "Nalmefene OR Baclofen OR Acamprosate OR Topiramate OR Naltrexone AND Alcohol" with the filter "Clinical Trial". The last update of the search was performed in August 2017.

Two reviewers (CP and RD) independently reviewed the titles and abstracts of all citations identified by the literature search. Two reviewers ( $\mathrm{CP}$ and $\mathrm{KH})$ independently examined the full text of relevant studies. All disagreements were resolved by consensus or consultation with another reviewer (FN). Unpublished studies were also searched for by consulting the registries of ClinicalTrials.gov, the Food and Drug Administration, and the EMA. The authors were contacted for further information as necessary. If no response was obtained after the first request, they were re-contacted.

\section{Assessment of methodological quality}

The risk of bias was assessed using the Cochrane Collaboration tool for assessing the risk of bias [26] for each RCT included in the study by two independent reviewers $(\mathrm{CP}$ and $\mathrm{KH})$. All disagreements were resolved by consensus or consultation with another reviewer (FN).

\section{Data collection}

A data extraction sheet, based on the Cochrane Handbook for Systematic Reviews of Interventions guidelines [27], was used to collect data from the RCTs. Data collection was performed by two reviewers (CP and $\mathrm{KH}$ ). All disagreements were resolved by consultation with a third reviewer (FN). Suspected duplicate studies were compared to avoid integrating data from several reports on the same study. For each included study, information concerning the characteristics of the study [year, country, publication status (i.e. published or unpublished), outcomes reported], trial participants [age, gender, medical condition (i.e. alcohol dependence or alcohol use disorders), abstinence before the beginning of the study, somatic, or psychiatric comorbidity], and intervention [treatments, dose, route of administration and duration, and psychological support] was extracted.

\section{Assessment of vibration of effects}

For each RCT, the treatment effect was calculated and expressed as the standardized mean difference (SMD, Hedges' $g$ ) for the different consumption outcomes (i.e. quantity of alcohol consumed, frequency of drinking, and abstinence) [28]. For abstinence, the log (odds ratio) was calculated and converted into SMD (Hedges' $g$ ) when the criterion reported in the study was the percentage of abstinent or relapsing subjects (i.e. binary outcomes) [28]. For direct comparisons, an estimate of the overall effect (summary measure) was calculated using both fixed and random effects models, with the inverse variance method. Indirect comparisons were performed using the graph theoretical method [29], a frequentist approach.

As a principal outcome, we explored the $\mathrm{VoE}$ of the indirect comparison between nalmefene and naltrexone. We computed the distribution of point estimates of effect sizes (ESs) and their corresponding $p$ values under various analytical scenarios defined by the combination of methodological choices. These methodological choices (detailed in Table 1) were based on different inclusion/exclusion criteria (i.e. medical condition, abstinence of patients before inclusion, gender, somatic and psychiatric comorbidity, psychological support, treatment administered and dose, treatment duration, outcome reported, publication status, or risk of bias) and different analytical models (i.e. fixed or random effects). A negative ES favoured nalmefene. Meta-analyses were considered to be statistically significant if the ES was associated with a $p$ value $<0.05$. The presence of a "Janus effect" was investigated by calculating the 1st and 99th percentiles of the distribution of the ES [16]. A Janus effect is defined as an ES which is in the opposite direction between the 1st and 99th percentiles of metaanalyses. It demonstrates the presence of substantial VoE [16]. In addition, we computed the distribution of the $I^{2}$ indices and the $p$ values of the test for heterogeneity (i.e. Cochran's $Q$ test) calculated for each scenario. Heterogeneity was considered to be statistically significant if the $p$ value of the $Q$ test was $<0.10$.

The same approach was used to analyse the secondary outcomes: VoE of the direct comparison of nalmefene to placebo and $\mathrm{VoE}$ of the direct comparison of naltrexone to placebo. For these analyses, a negative ES favoured the experimental treatment (i.e. nalmefene or naltrexone).

All analyses were performed using $\mathrm{R}$ [30] and the metagen [31] and netmeta [32] libraries. The results are presented according to the Preferred Reporting Items for Systematic Reviews and Meta-Analyses (PRISMA) format [33] and its extension for network meta-analyses [34]. The data and code are shared on the Open Science Framework (available at https://osf.io/skv2h/).

\section{Changes to the initial protocol}

As stated a priori in the protocol, we expected that several scenarios would not be feasible, depending on 
Table 1 Definition of the different methodological choices and number of possible analytical scenarios

\begin{tabular}{|c|c|c|}
\hline Category & Criteria & Number of possibilities \\
\hline Medical condition & $\begin{array}{l}\text { Inclusion of all studies (AUDs and/or AD) } \\
\text { Exclusion of studies including patients with AUDs }\end{array}$ & 2 \\
\hline Abstinence $^{\dagger}$ & $\begin{array}{l}\text { Inclusion of all studies (abstinent or non-abstinent patients) } \\
\text { Exclusion of studies requiring a minimum period of abstinence of } 5 \text { days } \\
\text { or more before the beginning of the study }\end{array}$ & 2 \\
\hline Gender & $\begin{array}{l}\text { Inclusion of all studies (mixed gender, males only or females only) } \\
\text { Exclusion of studies with males or females only }\end{array}$ & 2 \\
\hline Somatic comorbidity & $\begin{array}{l}\text { Inclusion of all studies (patients with or without systematic somatic } \\
\text { comorbidities) } \\
\text { Exclusion of studies on patients with systematic somatic comorbidities } \\
\text { (e.g. studies on patients with HIV) }\end{array}$ & 2 \\
\hline Psychiatric comorbidity & $\begin{array}{l}\text { Inclusion of all studies (patients with or without systematic psychiatric } \\
\text { comorbidities) } \\
\text { Exclusion of studies on patients with systematic psychiatric comorbidities } \\
\text { (e.g. studies on depressed patients) }\end{array}$ & 2 \\
\hline Psychological support & $\begin{array}{l}\text { Inclusion of all studies (with or without psychological intervention) } \\
\text { Exclusion of studies with no psychological intervention }\end{array}$ & 2 \\
\hline Treatment and dose & $\begin{array}{l}\text { Only approved dose and route of administration } \\
\text { Approved dose and route of administration OR closest dose to the } \\
\text { approved dose } \\
\text { Maximum dose tested }\end{array}$ & 3 \\
\hline Treatment duration & $\begin{array}{l}\text { Inclusion of all studies, regardless of treatment duration } \\
\text { Exclusion of studies with a treatment duration of less than } 12 \text { weeks }\end{array}$ & 2 \\
\hline Outcome $^{\ddagger}$ & $\begin{array}{l}\text { Quantity of alcohol consumed* } \\
\text { Frequency of drinking } \\
\text { Abstinence }^{* * *}\end{array}$ & 3 \\
\hline Publication & $\begin{array}{l}\text { Published and unpublished studies (e.g. study reports, ClinicalTrials.gov) } \\
\text { Exclusion of unpublished studies }\end{array}$ & 2 \\
\hline Risk of bias & $\begin{array}{l}\text { Inclusion of all studies, regardless of the risk of selective outcome } \\
\text { reporting } \\
\text { Exclusion of studies with a high risk of selective outcome reporting }\end{array}$ & 2 \\
\hline Analysis & $\begin{array}{l}\text { Fixed effect model } \\
\text { Random effect model }\end{array}$ & 2 \\
\hline Total of possible combinations & & 9216 \\
\hline
\end{tabular}

${ }^{\dagger}$ The choice of the 5-day cut-off was based on our previous meta-analysis [25]

${ }^{\ddagger}$ If there were several outcomes for quantity consumed, frequency of drinking, or abstinence reported in the same study, only one criterion of each type was collected

*Outcomes for the quantity of alcohol consumed were extracted in this order of preference: (1) total alcohol consumption, (2) number of drinks per day, (3) number of drinks per drinking day, and (4) alcohol consumption per drinking day

**Frequency of drinking outcomes was extracted in this order of preference: (1) number of heavy drinking days, (2) percentage of heavy drinking days, and (3) percentage of drinking days

***Abstinence outcomes were extracted in this order of preference: (1) number of abstinent days, (2) percentage of abstinent days, (3) percentage of abstinent subjects, and (4) percentage of relapsing subjects

data availability, and some were therefore modified. Although we initially planned to collect only the consumption outcomes to assess $\operatorname{VoE}$ (i.e. quantity of alcohol consumed and frequency of drinking), we finally decided to extract abstinence outcomes as well, as many RCTs that assessed the efficacy of naltrexone for post-withdrawal maintenance of abstinence only reported extractable data on abstinence, and not consumption outcomes. Introduction of this new choice made it difficult to impute missing data at the patient level using imputation methods, and we suppressed the analytical scenario for which the meta-analyses were performed with imputed data. We also planned to conduct meta- analyses with all RCTs versus RCTs with treatment durations of 5 months or more. We changed the cut-off to 3 months as there were few RCTs with treatment durations of 5 months or more (especially for naltrexone trials). Furthermore, the impact of the risk of bias on the treatment effect was only assessed for the risk of selective outcome reporting and not the risk of incomplete outcome data, as almost all included RCTs were at high risk of incomplete outcome data. Finally, the VoE was not assessed according to patient age, language of publication, or subgroup analysis on patients with a high risk of drinking, because no RCT including minor patients was included in the study; all studies were published in 
English, except one (published in Portuguese but still included in the analyses even if only studies in English, French, and Spanish were to be retrieved initially); and no extractable data from the subgroup analyses on patients with a high risk of drinking were identified for the naltrexone studies. As part of the peer review process, we decided to perform a sensitivity analysis excluding meta-analyses with $I^{2}>25 \%$ and based on a fixed effect model, because these combinations could be considered inappropriate to do.

\section{Results}

After adjusting for duplicates, we identified a total of 2001 citations. After the first round of selection based on the titles and abstracts, the full texts of 151 articles were assessed for eligibility. We excluded 71 papers, and 20 articles provided no data with which to calculate an effect size on any relevant outcomes. References of the articles excluded after the review of the full text are listed in Additional file 1: References S1. A flowchart detailing the study selection process is shown in Fig. 1.

\section{Study characteristics and risk of bias within studies}

Nine nalmefene versus placebo RCTs [35-41] and 51 naltrexone versus placebo RCTs [42-89] were considered eligible for the analyses. No study provided a direct comparison between nalmefene and naltrexone. The main characteristics of the included studies are summarized in Additional file 1: Table S1. The assessment of the risk of bias is reported in Additional file 1: Figure S1. One article was published in Portuguese [50]. Several studies were unpublished: data of 2 unpublished nalmefene RCTs (CPH-101-0399, CPH-101-0701) were provided by access to the document service of the EMA, and data of 3 naltrexone RCTs were recovered from ClinicalTrials.gov (NCT00667875, NCT01625091, and NCT00302133). No study was conducted on minor patients.

\section{Vibration of effects}

The distribution of the studies according to each methodological choice is presented in Table 2. We performed 9216 overlapping meta-analyses for the indirect comparison of nalmefene to naltrexone (resulting in 3856 different RCT combinations), the direct comparison of nalmefene to placebo (resulting in 86 different RCT combinations), and the direct comparison of naltrexone to placebo (resulting in 1988 different RCT combinations).

\section{Main analysis}

A median of 16 RCTs [interquartile range (IQR) $=12-21$ ] were included in the meta-analyses for the indirect comparison (Fig. 1). The distribution of the ES ranged from -0.37 to 0.31 , with a median of -0.04 . The ES was negative for the 1st percentile $(-0.29)$ and positive for the 99th percentile (0.29) (Fig. 2), indicating the presence of a Janus effect, with some meta-analyses showing a statistically significant superiority of nalmefene over naltrexone, whereas others showed the opposite effect. A total of $7.1 \%$ $(425 / 5961)$ of the meta-analyses with a negative ES (i.e. in favour of nalmefene) and $18.9 \%(616 / 3255)$ of the metaanalyses with a positive ES (i.e. in favour of naltrexone) were statistically significant $(p<0.05)$. An example of 2 meta-analyses with contradictory results is presented in Additional file 1: Table S2-S3. Concerning heterogeneity, the median of the $I^{2}$ index was $14 \%$ (IQR $=0-42 \%$ ), and the $p$ value of Cochran's $Q$ test was $<0.10$ for $31.4 \%$ (2896/9216) of the meta-analyses (Additional file 1: Figure S2). A similar VoE was found in the sensitivity analysis excluding the meta-analyses with $I^{2}>25 \%$ and based on a fixed effect model, with a negative ES for the 1st percentile $(-0.28)$ and a positive ES for the 99th percentile (0.30) (Additional file 1: Figure S3).

\section{Secondary analyses}

None of the meta-analyses performed on nalmefene RCTs favoured the placebo. The ES ranged from -0.25 to -0.06 , with a median of -0.19 . The ES was negative for both the 1st percentile and 99th percentiles $(-0.25$ and -0.06), and there was no Janus effect (Fig. 3). A total of $67.4 \%(6208 / 9216)$ of the meta-analyses were statistically significant. The heterogeneity was generally small (median of $I^{2}=0 \%, \mathrm{IQR}=0-25 \%$ ) (Additional file 1 : Figure S4), and the $p$ value of Cochran's $Q$ test was $<0.10$ for $7.6 \%(704 / 9216)$ of the meta-analyses.

The meta-analyses performed on naltrexone versus placebo RCTs provided ESs ranging from -0.38 to 0.16 , with a median of -0.16 . Although the ESs favouring placebo over naltrexone never reached statistical significance, there was a Janus effect: the ES was in opposite directions between the 1st $(E S=-0.37)$ and 99th percentiles $(E S=0.09)$ of the meta-analyses (Fig. 4). Only $6.5 \%(602 / 9216)$ of the meta-analyses were associated with a positive ES. Heterogeneity (median of $I^{2}=23 \%$, IQR $=3-48 \%$ ) (Additional file 1: Figure S5) was higher than for direct comparisons of nalmefene to placebo. The $p$ value of Cochran's $Q$ test was $<0.10$ for $34.9 \%$ $(3218 / 9216)$ of the meta-analyses.

\section{Discussion}

\section{Statement of principal findings}

VoE is a standardized method that can be used in any meta-analysis to systematically evaluate the breadth and divergence of the results, depending on the choices made in the selection of studies, based on various criteria, and the analytical model used. As a case study, we show extensive $\mathrm{VoE}$ in an indirect comparison of 


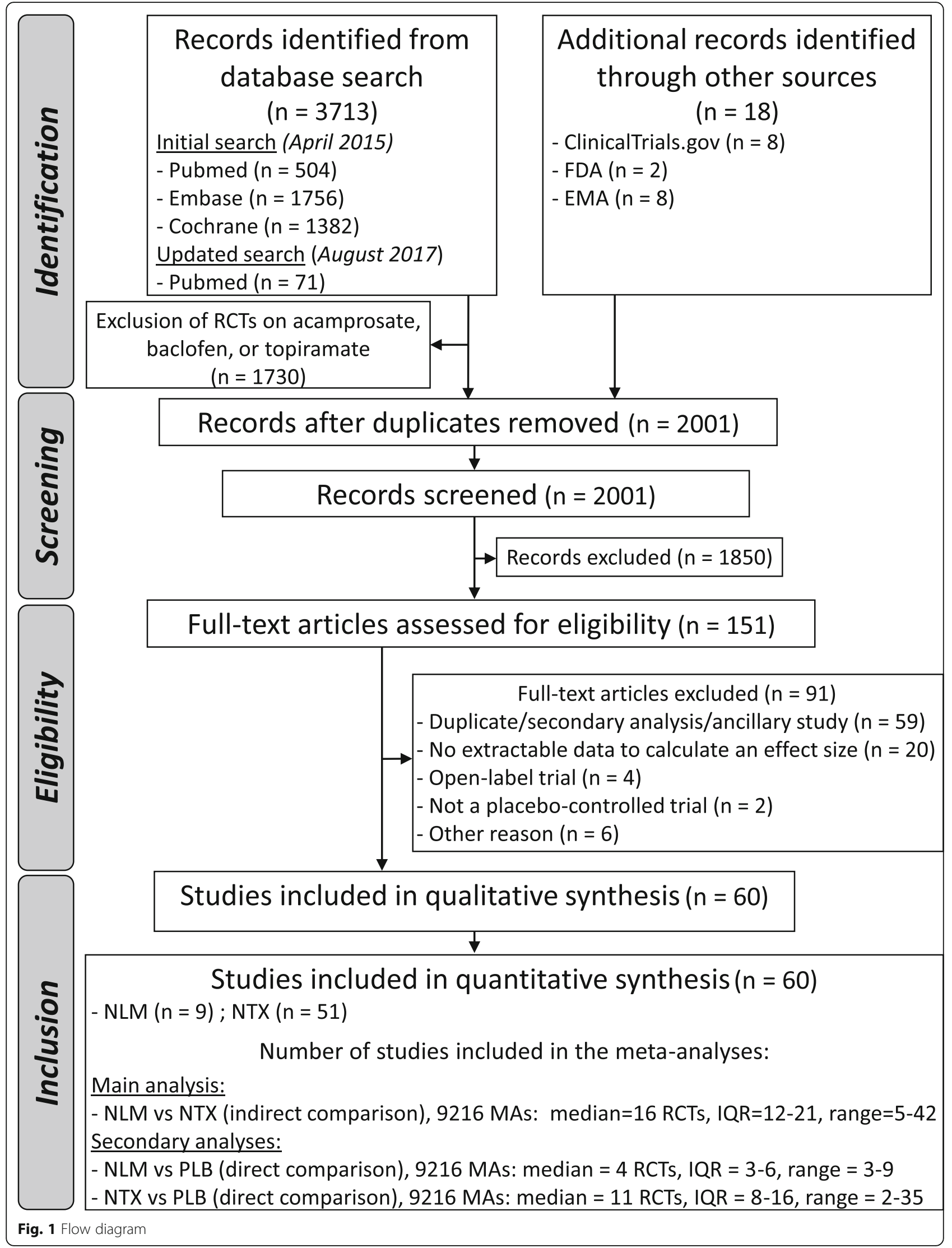


Table 2 Distribution of the studies according to each possible methodological choice

\begin{tabular}{|c|c|c|}
\hline Category & $\begin{array}{l}\text { Nalmefene } \\
(n=9)\end{array}$ & $\begin{array}{l}\text { Naltrexone } \\
(n=51)\end{array}$ \\
\hline \multicolumn{3}{|l|}{ Medical condition } \\
\hline AD only & $6(66.7 \%)$ & $33(64.7 \%)$ \\
\hline AUDs & $3(33.3 \%)$ & $18(35.3 \%)$ \\
\hline \multicolumn{3}{|l|}{ Abstinence } \\
\hline$<5$ days & $9(100 \%)$ & $34(66.7 \%)$ \\
\hline$\geq 5$ days & $0(0.0 \%)$ & $17(33.3 \%)$ \\
\hline \multicolumn{3}{|l|}{ Gender } \\
\hline Mixed & $9(100 \%)$ & $39(76.5 \%)$ \\
\hline Males or females only & $0(0.0 \%)$ & $12(23.5 \%)$ \\
\hline \multicolumn{3}{|l|}{ Systematic somatic comorbidity } \\
\hline No & $9(100 \%)$ & $48(94.1 \%)$ \\
\hline Yes & $0(0.0 \%)$ & $3(5.9 \%)$ \\
\hline \multicolumn{3}{|l|}{ Systematic psychiatric comorbidity } \\
\hline No & $9(100 \%)$ & $39(76.4 \%)$ \\
\hline Yes & $0(0.0 \%)$ & $12(23.5 \%)$ \\
\hline \multicolumn{3}{|l|}{ Psychological support } \\
\hline No & $1(11.1 \%)$ & $4(7.8 \%)$ \\
\hline Yes & $8(88.9 \%)$ & $47(92.2 \%)$ \\
\hline \multicolumn{3}{|l|}{ Treatment and dose } \\
\hline \multicolumn{3}{|l|}{ Approved dose and route of administration } \\
\hline No & $6(66.7 \%)$ & $14(27.5 \%)$ \\
\hline Yes & $3(33.3 \%)$ & $37(72.5 \%)$ \\
\hline \multicolumn{3}{|l|}{ Maximum dose tested } \\
\hline No & $8(88.9 \%)$ & $50(98.0 \%)$ \\
\hline Yes & $1(11.1 \%)$ & $1(2.0 \%)$ \\
\hline \multicolumn{3}{|l|}{ Treatment duration } \\
\hline$\geq 12$ weeks & $9(100 \%)$ & $41(80.4 \%)$ \\
\hline$<12$ weeks & $0(0.0 \%)$ & $10(19.6 \%)$ \\
\hline \multicolumn{3}{|l|}{ Outcome reported } \\
\hline \multicolumn{3}{|l|}{ Quantity of alcohol consumed } \\
\hline No & $0(0.0 \%)$ & $21(41.2 \%)$ \\
\hline Yes & $9(100 \%)$ & $30(58.8 \%)$ \\
\hline \multicolumn{3}{|l|}{ Frequency of drinking } \\
\hline No & $2(22.2 \%)$ & $16(31.4 \%)$ \\
\hline Yes & $7(77.8 \%)$ & $35(68.6 \%)$ \\
\hline \multicolumn{3}{|l|}{ Abstinence } \\
\hline No & $0(0.0 \%)$ & $18(35.3 \%)$ \\
\hline Yes & $9(100 \%)$ & $33(64.7 \%)$ \\
\hline \multicolumn{3}{|l|}{ Publication } \\
\hline Published & $7(77.8 \%)$ & $48(94.1 \%)$ \\
\hline Unpublished & $2(22.2 \%)$ & $3(5.9 \%)$ \\
\hline \multicolumn{3}{|l|}{ Risk of bias } \\
\hline High risk of selective outcome reporting & $2(22.2 \%)$ & $5(9.8 \%)$ \\
\hline Unclear or low risk of selective outcome reporting & $7(77.8 \%)$ & $46(90.2 \%)$ \\
\hline
\end{tabular}

Numbers are presented with their corresponding percentage $A D$ alcohol dependence, $A U D$ alcohol use disorder nalmefene to naltrexone, leading to contradictory results. Although most combinations yielded no evidence of a difference, some meta-analyses showed superiority of nalmefene, whereas others showed superiority of naltrexone. These two compounds have many similarities, and it is unlikely to expect a genuine difference [19]. When we considered direct comparisons against placebo, we observed less $\mathrm{VoE}$ for nalmefene than for naltrexone. Nalmefene is the most recent treatment option and has been the subject of two distinct but somewhat homogeneous development programmes [90], resulting in several studies with a similar design. In contrast, naltrexone is an older option with a myriad of pre- and post-approval RCTs conducted in very different settings.

\section{Strengths and weaknesses of this study}

We recommend that a list of all possible major options should be made first when examining $\mathrm{VoE}$ in a metaanalysis. Nevertheless, we acknowledge that even the construction of such a list may itself be subject to unavoidable subjectivity. All the methodological choices we made to assess VoE in our case study corresponded to the criteria we considered to be easily "gameable". Several may be clinically relevant, such as the exclusion of studies on abstinent patients or those on patients with a somatic or psychiatric comorbidity. Others are related to the literature search, such as the retrieval of unpublished studies. Most meta-analyses have difficulty unearthing unpublished studies, and publication bias [91] may affect the treatment ranking in indirect metaanalyses [92]. The relevance of other combinations may be debatable. For example, the use of fixed effect models in case of between-study heterogeneity is not considered statistically valid and would not be considered for publication. However, our sensitivity analysis that excluded meta-analyses that were considered inappropriate to do still found some VoE. It is difficult and subjective to judge the appropriateness of different combinations; the two illustrative examples (Additional file 1: Table S2-S3) demonstrate that contradictory meta-analyses are not necessarily inappropriate, per se. It is also likely that some datasets we combined violated the similarity assumption required for indirect comparisons. Dissimilar study results due to treatment effect modifiers may have led to some of the VoE we observed. In theory, positive and negative results from multiple meta-analyses are not necessarily contradictory if the inclusion criteria are so different that the results would apply to different research questions. However, in practice, the identification of treatment effect modifiers is very challenging [93], and it is sometimes very difficult and subjective to make a clear judgement on how much different methodological choices really define different research questions. Here, we tried to pre-emptively retain the choices that 


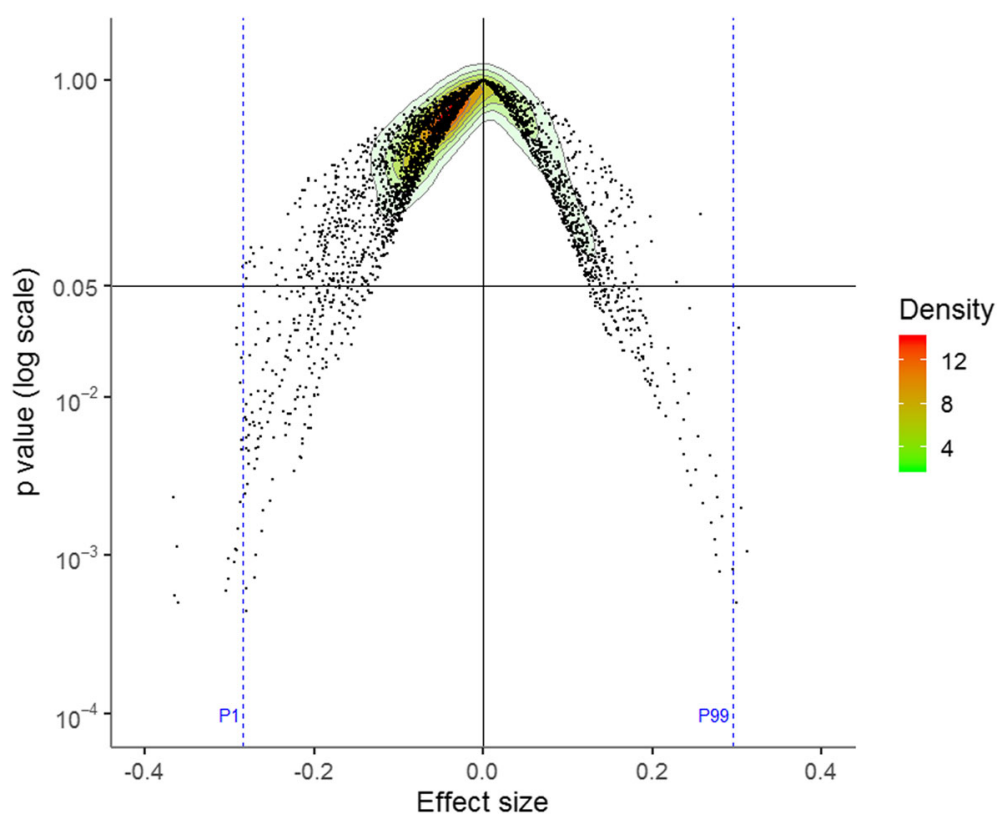

Fig. 2 Vibration of effects for the indirect comparison of nalmefene to naltrexone. A negative effect size favours nalmefene, whereas a positive effect size favours naltrexone. The points represent the meta-analyses. The colours represent the densities

would not have altered the research question, and we minimized the possibility of making mutually exclusive methodological choices. Moreover, our study was based on a relatively limited number of methodological choices, which may underestimate the whole set of alternative scenarios, and the $\mathrm{VoE}$ could have been even greater. For example, we could have studied the VoE depending on whether the indirect comparisons were made using a Bayesian approach or a frequentist approach. Another potential source of $\mathrm{VoE}$ was not investigated, namely the choice of the source from which data from a study are extracted (e.g. published articles, study

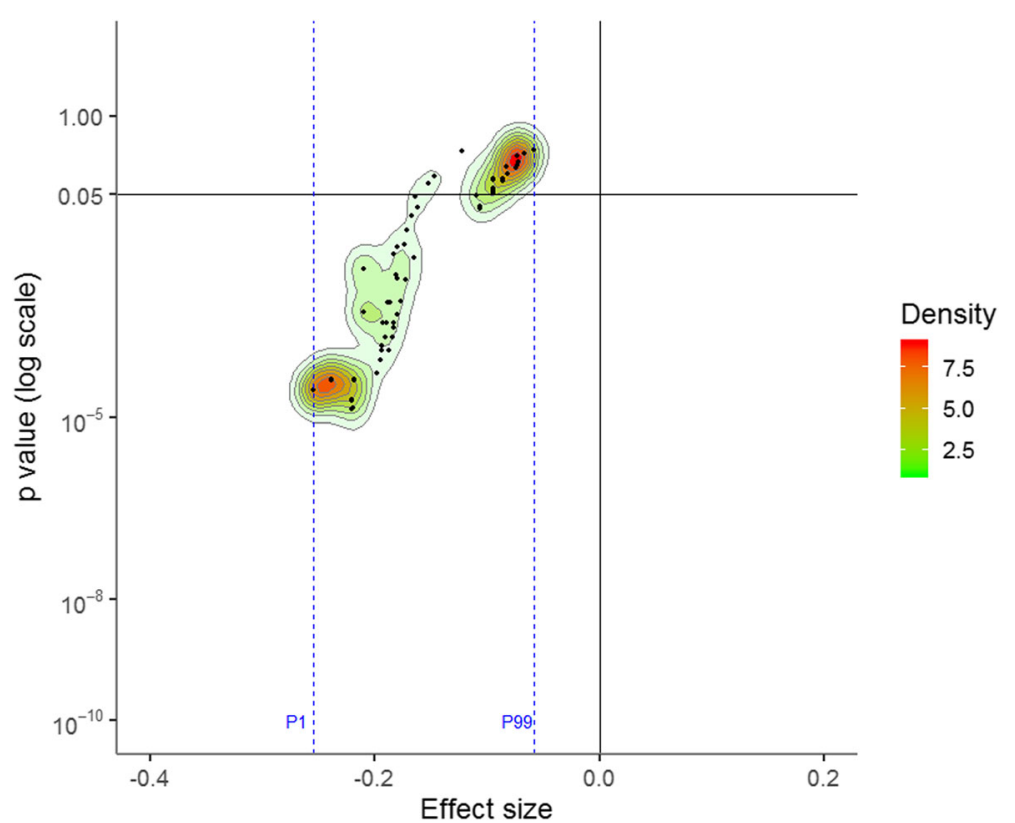

Fig. 3 Vibration of effects for the direct comparison of nalmefene to placebo. A negative effect size favours nalmefene, whereas a positive effect size favours the placebo. The points represent the meta-analyses. The colours represent the densities 


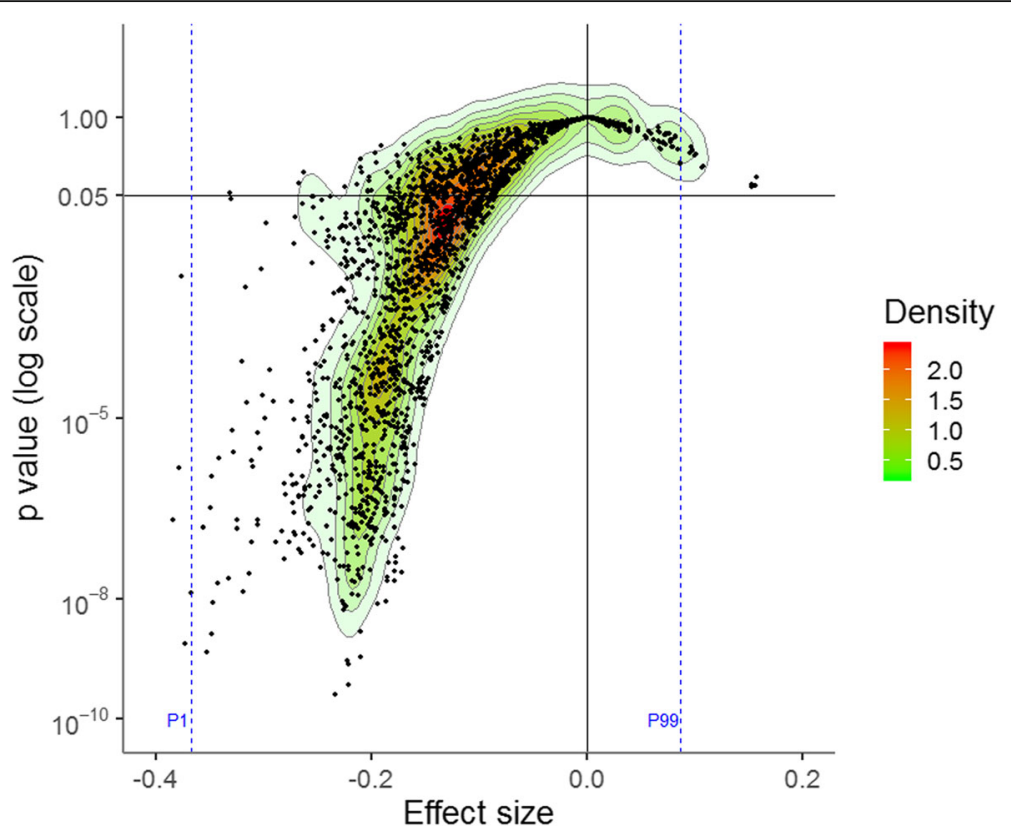

Fig. 4 Vibration of effects for the direct comparison of naltrexone to placebo. A negative effect size favours naltrexone, whereas a positive effect size favours the placebo. The points represent the meta-analyses. The colours represent the densities

reports, ClinicalTrials.gov). It is possible that in other contexts, such as meta-analyses exploring drug safety, non-randomized studies may be included and add even more VoE, especially due to possible bias (e.g. indication bias) in the primary studies.

Indirect comparison meta-analyses may yield less $\mathrm{VoE}$ in other, less controversial fields, in which the results of studies are more homogeneous. Conversely, VoE may be more prominent in complex, heterogeneous meta-analyses, such as that of large networks with prominent inconsistency [94]. For example, it has been shown in network meta-analyses that even the consideration of which nodes are eligible and/or whether a placebo should be considered can already yield very different results [95]. In addition, some of the contradictory meta-analyses that are generated in the VoE exercise may not pass peer review, receive harsh criticism for their choices, or even be retracted after publication, as for a meta-analysis of acupuncture [96]. Therefore, we recommend that the choice of factors to consider in the $\mathrm{VoE}$ analyses should be realistic.

\section{Perspectives}

VoE has already been described in the field of observational epidemiology $[16,97]$, but has been less explored in meta-analyses. Nevertheless, a previous study showed that it is possible to manipulate the effect sizes based on the discrepancies among multiple data sources (papers, clinical study reports, individual patient data) [98]. In this study, the overall result of the meta-analyses performed to assess the ES of gabapentin for the treatment of pain intensity switched from effective to ineffective and the overall result for the treatment of depression with quetiapine from medium to small, depending on the data source. In our study, cherry-picking results from each included RCT may have introduced VoE without changing either the trial inclusion criteria or the methods of meta-analysis.

There is a large body of literature on discordant metaanalyses. Indeed, the first widely known meta-analyses in medicine were probably those performed by opposing teams in the 1970s that found opposite results on the risk of gastrointestinal bleeding from steroids. Over the years, debate has often arisen within specific topics in which two or more meta-analyses on seemingly the same question reached different conclusions [6-13]. Discussion of the main reasons put forth for the discrepancy for each case, with careful clinical reasoning, is likely to continue being useful. VoE analysis offers a complementary systematic approach to evaluate the potential for a discrepancy in any meta-analysis, including large-scale meta-analyses. VoE offers a more generalized view of sensitivity analyses. Typically, some level of sensitivity analysis is commonly performed. For example, many/most meta-analyses may present side-by-side fixed and random effects models, as performed in our case study. Additional methodological choices may generate more sensitivity analyses related to the choice of outcome measure, handling of missing data, correction for potential bias, interdependence, etc. [99-102]. Sensitivity 
analyses based on clinical characteristics are also common, but usually, only a few such analyses are reported, if at all.

Although the evaluation of $\mathrm{VoE}$ is generally systematic and involves more extensive analysis than the sporadic sensitivity analyses typically performed in past metaanalyses, it still requires a priori determination of the factors considered to be most relevant for making choices in the conduct of a meta-analysis. In this respect, it is not as clinically agnostic as the all-subset method, in which all possible meta-analyses of all possible subsets of studies are explored for a given set of studies to be meta-analysed [103]. This method runs into computational difficulties with large meta-analyses. For example, application of the all-subset method for VoE in the current case study, with 51 and 9 trials, would result in $2^{51}$ and $2^{9}$ possible subsets, respectively, with $2^{51+9}=2^{60}$ different indirect meta-analyses, i.e. 1,152,921,504,606, 846,976 different indirect comparison meta-analyses to be performed, a number that is computationally absurd to explore and not clinically relevant.

Systematic reviews and meta-analyses (including indirect comparisons and more complex networks) are often considered to offer the highest level of evidence [104]. These studies have become so influential that they can shape guidelines and change clinical practice. However, their use has reached epidemic proportions, and published meta-analyses [104] and network meta-analyses [5] are subject to extensive overlap and potential redundancy. It has been argued that these studies could be used as key marketing tools when there are strong conflicts concerning which results are preferable to highlight. For example, numerous meta-analyses of antidepressants authored by or linked to the industry have been described previously [105]. Industry-linked studies almost never report any caveats about antidepressants in their abstracts. Conversely, it is a common practice in the industry to commission network meta-analyses to professional contracting companies, and thus, most are not registered a priori or published. A veto from industry is the most commonly stated reason for not having a publication plan for network meta-analyses [106]. VoE is a method that could be used to highlight selective reporting and controversial results.

The extent of redundant meta-analyses and wasted efforts may be reduced with protocol pre-registration, for example, with the PROSPERO database [107]. Nevertheless, registration does not provide the same guarantees for meta-analyses as for RCTs. For RCTs, registration is prospectively performed, before enrolment of the first patient. Conversely, meta-analyses are almost always retrospective (i.e. planned after the individual studies are completed), and even registration cannot prevent "cherry picking" of some methodological choices based on preliminary analyses of the existing data. The development of prospective meta-analyses could avoid these pitfalls, as in such meta-analyses, studies are identified, evaluated, and determined to be eligible before the results of any of the studies become known [27].

Even if meta-analysis protocols are thoroughly and thoughtfully designed, a number of analytical and eligibility choices still need to be made and many may be subjective. An applicable safeguard could be the a priori reviewing of protocols by independent experts and comities that might prevent meta-analyses of being gameable. In addition, VoE allows a systematic exploration of the influence of analytical and eligibility choices on the treatment effect. It appears to be a tool that is worth developing in different contexts, such as head-tohead, network, and individual patient data meta-analyses. Systematically exploring VoE in a large set of metaanalyses may provide a better sense of its relevance.

\section{Conclusions}

Multiplication of overlapping meta-analyses may more frequently yield contradictory results that are difficult to interpret. Controversial and conflicting meta-analyses can result in the loss of credibility in the eyes of patients, the medical community, and the policy-makers. Efforts must be made to improve the reproducibility and transparency [108] of research and minimize $\mathrm{VoE}$, whenever possible. In most circumstances, the most feasible approach would be to determine the magnitude of the potential risk of obtaining discrepant results, for which VoE could be a useful tool.

\section{Additional file}

\begin{abstract}
Additional file 1: References S1. References of articles excluded after review of full-texts. Table S1. Main characteristics of the included studies. Figure S1. Quality evaluation of studies included according to the Cochrane Collaboration tool for assessing risk of bias. Table S2. Analytica scenario resulting in superiority of nalmefene over naltrexone. Table S3. Analytical scenario resulting in superiority of naltrexone over nalmefene. Figure S2. Heterogeneity of the indirect comparison: nalmefene versus naltrexone. Figure S3. Sensitivity analysis excluding meta-analyses with $P^{2}>25 \%$ and based on a fixed effect model for the indirect comparison between nalmefene and naltrexone. Figure S4. Heterogeneity of the direct comparison: nalmefene versus placebo. Figure S5. Heterogeneity of the direct comparison: naltrexone versus placebo. Checklist S1. PRISMA checklist. (PDF 562 kb)
\end{abstract}

\section{Abbreviations}

AD: Alcohol dependence; EMA: European Medicines Agency; ES: Effect size; $\mathrm{RCT}$ : Randomized control trial; SMD: Standardized mean difference; VoE: Vibration of effects

\section{Acknowledgements}

We would like to thank all the authors who agreed to provide additional information concerning their studies. 


\section{Authors' contributions}

$\mathrm{FN}, \mathrm{CP}$, and JPAl conceived and designed the experiments. $\mathrm{CP}, \mathrm{KH}, \mathrm{RD}$, and FN performed the experiments. $\mathrm{CP}$ and $\mathrm{KH}$ analysed the data. $\mathrm{CP}$ and FN wrote the first draft of the manuscript. $\mathrm{KH}$ and JPAl contributed to the writing of the manuscript. CP, KH, RD, BL, JPAl and FN agreed with the results and conclusions of the manuscript. All authors read and approved the final manuscript.

\section{Funding}

The study was funded by Rennes CHU (CORECT; Comité de la Recherche Clinique et Translationnelle). The funder of the study had no role in the study design, data collection, data analysis, data interpretation, or writing of the report. The corresponding author had full access to all data in the study and had final responsibility for the decision to submit for publication.

\section{Availability of data and materials}

The data and code are shared on the Open Science Framework (available at https://osf.io/skv2h/s.

\section{Ethics approval and consent to participate}

Not applicable.

\section{Consent for publication}

Not applicable.

\section{Competing interests}

The authors declare that they have no competing interests. All authors have completed the Unified Competing Interest form at http://www.icmje.org/ coi_disclosure.pdf (available on request from the corresponding author) and declare that (1) no authors have support from any company for the submitted work; (2) CP, KH, RD, BL, Jl, and FN have had no relationships with any company that might have an interest in the submitted work in the previous 3 years; (3) no author's spouse, partner, or children have any financial relationships that could be relevant to the submitted work; and (4) none of the authors has any non-financial interests that could be relevant to the submitted work

\section{Author details}

${ }^{1}$ Centre d'Investigation Clinique INSERM 1414, Hôpital de Pontchaillou, 2 rue Henri le Guilloux, 35033 Rennes cedex 9, France. ${ }^{2}$ Department of Biostatistics, Institut de Cancérologie de l'Ouest Centre René-Gauducheau, Saint-Herblain, France. ${ }^{3}$ Department of Epidemiology and Biostatistics and Clinical Research, Assistance Publique-Hôpitaux de Paris, Hôpital Bichat Claude Bernard, Paris, France. ${ }^{4}$ Inserm, CIC-EC 1425, Hôpital Bichat Claude Bernard, Paris, France. ${ }^{5}$ Fondation Saint Jean de Dieu, Centre Hospitalier Dinan/St Brieuc, Dinan, France. ${ }^{6}$ Department of Biological and Clinical Pharmacology and Pharmacovigilance, Rennes University Hospital, Rennes, France. 'Laboratory of Experimental and Clinical Pharmacology, Rennes 1 University, Rennes, France. ${ }^{8}$ Meta-Research Innovation Center at Stanford (METRICS), Stanford University, Stanford, CA, USA. 'Departments of Medicine, of Health Research and Policy, Biomedical Data Science, and Statistics, Stanford University, Stanford, CA, USA.

\section{Received: 10 May 2019 Accepted: 14 August 2019} Published online: 16 September 2019

\section{References}

1. Leucht S, Chaimani A, Cipriani AS, Davis JM, Furukawa TA, Salanti G. Network meta-analyses should be the highest level of evidence in treatment guidelines. Eur Arch Psychiatry Clin Neurosci. 2016;266:477-80.

2. Ioannidis JPA. The mass production of redundant, misleading, and conflicted systematic reviews and meta-analyses. Milbank Q. 2016:94:485-514.

3. loannidis JPA. Meta-research: the art of getting it wrong. Res Synth Methods. 2010;1:169-84

4. Siontis KC, loannidis JPA. Replication, duplication, and waste in a quarter million systematic reviews and meta-analyses. Circ Cardiovasc Qual Outcomes. 2018;11:e005212.

5. Naudet F, Schuit E, loannidis JPA. Overlapping network meta-analyses on the same topic: survey of published studies. Int J Epidemiol. 2017; 46:1999-2008
6. Cook DJ, Reeve BK, Guyatt GH, Heyland DK, Griffith LE, Buckingham L, et al. Stress ulcer prophylaxis in critically ill patients. Resolving discordant meta-analyses. JAMA. 1996;275:308-14.

7. Teehan GS, Liangos O, Lau J, Levey AS, Pereira BJG, Jaber BL. Dialysis membrane and modality in acute renal failure: understanding discordant meta-analyses. Semin Dial. 2003;16:356-60.

8. Vamvakas EC. Why have meta-analyses of randomized controlled trials of the association between non-white-blood-cell-reduced allogeneic blood transfusion and postoperative infection produced discordant results? Vox Sang. 2007;93:196-207.

9. Druyts E, Thorlund K, Humphreys S, Lion M, Cooper CL, Mills EJ. Interpreting discordant indirect and multiple treatment comparison meta-analyses: an evaluation of direct acting antivirals for chronic hepatitis $\mathrm{C}$ infection. Clin Epidemiol. 2013;5:173-83.

10. Susantitaphong P, Jaber BL. Understanding discordant meta-analyses of convective dialytic therapies for chronic kidney failure. Am J Kidney Dis Off J Natl Kidney Found. 2014;63:888-91.

11. Osnabrugge RL, Head SJ, Zijlstra F, ten Berg JM, Hunink MG, Kappetein AP, et al. A systematic review and critical assessment of 11 discordant metaanalyses on reduced-function CYP2C19 genotype and risk of adverse clinical outcomes in clopidogrel users. Genet Med Off J Am Coll Med Genet. 2015;17:3-11.

12. Lucenteforte E, Moja L, Pecoraro V, Conti AA, Conti A, Crudeli E, et al. Discordances originated by multiple meta-analyses on interventions for myocardial infarction: a systematic review. J Clin Epidemiol. 2015;68:246-56.

13. Bolland MJ, Grey A. A case study of discordant overlapping meta-analyses: vitamin d supplements and fracture. PLoS One. 2014;9:e115934.

14. Dechartres A, Altman DG, Trinquart L, Boutron I, Ravaud P. Association between analytic strategy and estimates of treatment outcomes in meta-analyses. JAMA. 2014;312:623-30.

15. Ioannidis JPA. Why most discovered true associations are inflated. Epidemio Camb Mass. 2008:19:640-8.

16. Patel CJ, Burford B, loannidis JPA. Assessment of vibration of effects due to model specification can demonstrate the instability of observational associations. J Clin Epidemiol. 2015;68:1046-58.

17. Serghiou S, Patel CJ, Tan YY, Koay P, loannidis JPA. Field-wide meta-analyses of observational associations can map selective availability of risk factors and the impact of model specifications. J Clin Epidemiol. 2016;71:58-67.

18. France $C P$, Gerak LR. Behavioral effects of 6-methylene naltrexone (nalmefene) in rhesus monkeys. J Pharmacol Exp Ther. 1994;270:992-9.

19. Swift RM. Naltrexone and nalmefene: any meaningful difference? Biol Psychiatry. 2013;73:700-1.

20. European Medicines Agency. Assessment report: Selincro—international non-proprietory name: nalmefene. EMA/78844/2013. 13 Dec 2012. Available at: http://www.ema.europa.eu/docs/en_GB/document_library/EPAR_Public_assessment_report/human/002583/WC500140326.pdf. Accessed 7 May 2018

21. Naudet F, Palpacuer C, Boussageon R, Laviolle B. Evaluation in alcohol use disorders - insights from the nalmefene experience. BMC Med. 2016;14:119.

22. Stafford N. German evaluation says new drug for alcohol dependence is no better than old one. BMJ. 2014;349:97544.

23. Soyka M, Friede M, Schnitker J. Comparing nalmefene and naltrexone in alcohol dependence: are there any differences? Results from an indirect meta-analysis. Pharmacopsychiatry. 2016;49:66-75.

24. Naudet F. Comparing nalmefene and naltrexone in alcohol dependence: is there a spin? Pharmacopsychiatry. 2016;49:260-1.

25. Palpacuer C, Duprez R, Huneau A, Locher C, Boussageon R, Laviolle B, et al. Pharmacologically controlled drinking in the treatment of alcohol dependence or alcohol use disorders: a systematic review with direct and network meta-analyses on nalmefene, naltrexone, acamprosate, baclofen and topiramate. Addiction. 2018;113:220-37.

26. Higgins JPT, Altman DG, Gøtzsche PC, Jüni P, Moher D, Oxman AD, et al. The Cochrane Collaboration's tool for assessing risk of bias in randomised trials. BMJ. 2011:343:d5928.

27. Higgins J. P. T., Green S., editors. Cochrane handbook for systematic reviews of interventions. Cochrane Collaboration. Version 5.1.0. Available at: http:// handbook-5-1.cochrane.org/ (Accessed 23 Aug 2017) (Archived at http:// www.webcitation.org/6sw9vRlcB).

28. Borenstein M, Hedges LV, Higgins JPT, Rothstein HR. References. In: Introduction to meta-analysis: Wiley; 2009. p. 409-14. https://doi.org/10.1 002/9780470743386.refs. 
29. Rücker G. Network meta-analysis, electrical networks and graph theory. Res Synth Methods. 2012;3:312-24.

30. R Development Core Team. R: a language and environment for statistical computing. Vienna: R Foundation for Statistical Computing; 2009.

31. Schwarzer G. Meta: general package for meta-analysis, version 3.6-0. 27May 2014. Available at: https://cran.r-project. org/web/packages/meta/ (Accessed 23 Aug 2017) (Archived at http://www.webcitation.org/6swAAXySQ).

32. Rücker G., Schwarzer G., Krahn U., König J. netmeta: network meta-analysis using frequentist methods. R package version 0.8-0, 2015. (Available at: https://cran.r-project. org/web/packages/netmeta/ (Accessed 23 Aug 2017) (Archived at http://www.webcitation.org/6swALYuOx).

33. Liberati A, Altman DG, Tetzlaff J, Mulrow C, Gøtzsche PC, loannidis JPA, et al. The PRISMA statement for reporting systematic reviews and meta-analyses of studies that evaluate health care interventions: explanation and elaboration. J Clin Epidemiol. 2009:62:e1-34.

34. Hutton B, Salanti G, Caldwell DM, Chaimani A, Schmid CH, Cameron C, et al. The PRISMA extension statement for reporting of systematic reviews incorporating network meta-analyses of health care interventions: checklist and explanations. Ann Intern Med. 2015;162:777-84.

35. Anton RF, Pettinati H, Zweben A, Kranzler HR, Johnson B, Bohn MJ, et al. A multi-site dose ranging study of nalmefene in the treatment of alcohol dependence. J Clin Psychopharmacol. 2004;24:421-8.

36. Gual A, He Y, Torup L, van den Brink W, Mann K. A randomised, doubleblind, placebo-controlled, efficacy study of nalmefene, as-needed use, in patients with alcohol dependence. Eur Neuropsychopharmacol. 2013:23:1432-42

37. Karhuvaara S, Simojoki K, Virta A, Rosberg M, Loyttyniemi E, Nurminen T, et al. Targeted nalmefene with simple medical management in the treatment of heavy drinkers: a randomized double-blind placebo-controlled multicenter study. Alcohol Clin Exp Res. 2007;31:1179-87.

38. Mann K, Bladstrom A, Torup L, Gual A, van den Brink W. Extending the treatment options in alcohol dependence: a randomized controlled study of as-needed nalmefene. Biol Psychiatry. 2013;73:706-13.

39. Mason BJ, Ritvo EC, Morgan RO, Salvato FR, Goldberg G, Welch B, et al. A double-blind, placebo-controlled pilot study to evaluate the efficacy and safety of oral nalmefene $\mathrm{HCl}$ for alcohol dependence. Alcohol Clin Exp Res. 1994;18:1162-7

40. Mason BJ, Salvato FR, Williams LD, Ritvo EC, Cutler RB. A double-blind, placebo-controlled study of oral nalmefene for alcohol dependence. Arch Gen Psychiatry. 1999;56:719-24.

41. Brink W, Sorensen P, Torup L, Mann K, Gual A. Long-term efficacy, tolerability and safety of nalmefene as-needed in patients with alcohol dependence: a 1-year, randomised controlled study. J Psychopharmacol Oxf Engl. 2014;28:733-44.

42. Ahmadi J, Babaeebeigi M, Maany I, Porter J, Mohagheghzadeh M, Ahmadi $\mathrm{N}$, et al. Naltrexone for alcohol-dependent patients. Ir J Med Sci. 2004;173:34-7.

43. Anton RF, Moak DH, Waid LR, Latham PK, Malcolm RJ, Dias JK. Naltrexone and cognitive behavioral therapy for the treatment of outpatient alcoholics: results of a placebo-controlled trial. Am J Psychiatry. 1999;156:1758-64.

44. Anton RF, Moak DH, Latham P, Waid LR, Myrick H, Voronin K, et al. Naltrexone combined with either cognitive behavioral or motivational enhancement therapy for alcohol dependence. J Clin Psychopharmacol. 2005;25:349-57.

45. Anton RF, O'Malley SS, Ciraulo DA, Cisler RA, Couper D, Donovan DM, et al. Combined pharmacotherapies and behavioral interventions for alcohol dependence: the COMBINE study: a randomized controlled trial. JAMA. 2006;295:2003-17.

46. Anton RF, Myrick H, Wright TM, Latham PK, Baros AM, Waid LR, et al. Gabapentin combined with naltrexone for the treatment of alcohol dependence. Am J Psychiatry. 2011;168:709-17.

47. Balldin J, Berglund M, Borg S, Mansson M, Bendtsen P, Franck J, et al. A 6-month controlled naltrexone study: combined effect with cognitive behavioral therapy in outpatient treatment of alcohol dependence. Alcohol Clin Exp Res. 2003;27:1142-9.

48. Baltieri DA, Daró FR, Ribeiro PL, Andrade AG. Comparing topiramate with naltrexone in the treatment of alcohol dependence. Addiction. 2008;103:2035-44.

49. Budzynski J, Rybakowski J, Swiatkowski M, Torlinski L, Klopocka M, Kosmowski W, et al. Naltrexone exerts a favourable effect on plasma lipids in abstinent patients with alcohol dependence. Alcohol Alcohol. 2000;35:91-7.

50. Castro LA, Laranjeira R. A double blind, randomized and placebo-controlled clinical trial with naltrexone and brief intervention in outpatient treatment of alcohol dependence. J Bras Psiquiatr. 2009;58:79-85.

51. Chick J, Anton R, Checinski K, Croop R, Drummond DC, Farmer R, et al. A multicentre, randomized, double-blind, placebo-controlled trial of naltrexone in the treatment of alcohol dependence or abuse. Alcohol Alcohol. 2000;35:587-93.

52. Cook RL, Weber KM, Mai D, Thoma K, Hu X, Brumback B, et al. Acceptability and feasibility of a randomized clinical trial of oral naltrexone vs. placebo for women living with HIV infection: study design challenges and pilot study results. Contemp Clin Trials. 2017;60:72-7.

53. Davidson D, Saha C, Scifres S, Fyffe J, O'Connor S, Selzer C. Naltrexone and brief counseling to reduce heavy drinking in hazardous drinkers. Addict Behav. 2004;29:1253-8

54. Foa EB, Yusko DA, McLean CP, Suvak MK, Bux DA Jr, Oslin D, et al. Concurrent naltrexone and prolonged exposure therapy for patients with comorbid alcohol dependence and PTSD: a randomized clinical trial. JAMA. 2013;310:488-95.

55. Fogaca MN, Santos-Galduroz RF, Eserian JK, Galduroz JC. The effects of polyunsaturated fatty acids in alcohol dependence treatment--a doubleblind, placebo-controlled pilot study. BMC Clin Pharmacol. 2011;11:10.

56. Fridberg DJ, Cao D, Grant JE, King AC. Naltrexone improves quit rates, attenuates smoking urge, and reduces alcohol use in heavy drinking smokers attempting to quit smoking. Alcohol Clin Exp Res. 2014;38:2622-9.

57. Garbutt JC, Kranzler HR, O'Malley SS, Gastfriend DR, Pettinati HM, Silverman $\mathrm{BL}$, et al. Efficacy and tolerability of long-acting injectable naltrexone for alcohol dependence: a randomized controlled trial. JAMA. 2005;293:1617-25.

58. Garbutt JC, Kampov-Polevoy AB, Kalka-Juhl LS, Gallop RJ. Association of the sweet-liking phenotype and craving for alcohol with the response to naltrexone treatment in alcohol dependence: a randomized clinical trial. JAMA Psychiatry. 2016;73:1056-63.

59. Gastpar M, Bonnet U, Boning J, Mann K, Schmidt LG, Soyka M, et al. Lack of efficacy of naltrexone in the prevention of alcohol relapse: results from a German multicenter study. J Clin Psychopharmacol. 2002;22:592-8.

60. Guardia J, Caso C, Arias F, Gual A, Sanahuja J, Ramirez M, et al. A doubleblind, placebo-controlled study of naltrexone in the treatment of alcoholdependence disorder: results from a multicenter clinical trial. Alcohol Clin Exp Res. 2002;26:1381-7.

61. Hersh D, Van Kirk JR, Kranzler HR. Naltrexone treatment of comorbid alcohol and cocaine use disorders. Psychopharmacol Berl. 1998;139:44-52.

62. Johnson BA, Ait-Daoud N, Aubin HJ, Van Den Brink W, Guzzetta R, Loewy J, et al. A pilot evaluation of the safety and tolerability of repeat dose administration of long-acting injectable naltrexone (Vivitrex) in patients with alcohol dependence. Alcohol Clin Exp Res. 2004;28:1356-61.

63. Killeen TK, Brady KT, Gold PB, Simpson KN, Faldowski RA, Tyson C, et al. Effectiveness of naltrexone in a community treatment program. Alcohol Clin Exp Res. 2004:28:1710-7.

64. Kranzler HR, Modesto-Lowe V, Van Kirk J. Naltrexone vs nefazodone for treatment of alcohol dependence. A placebo-controlled trial. Neuropsychopharmacology. 2000;22:493-503.

65. Kranzler HR, Wesson DR, Billot L. Naltrexone depot for treatment of alcohol dependence: a multicenter, randomized, placebo-controlled clinical trial. Alcohol Clin Exp Res. 2004;28:1051-9.

66. Kranzler HR, Tennen H, Armeli S, Chan G, Covault J, Arias A, et al. Targeted naltrexone for problem drinkers. J Clin Psychopharmacol. 2009;29:350-7.

67. Krystal JH, Cramer JA, Krol WF, Kirk GF, Rosenheck RA. Naltrexone in the treatment of alcohol dependence. N Engl J Med. 2001;345:1734-9.

68. Latt NC, Jurd S, Houseman J, Wutzke SE. Naltrexone in alcohol dependence: a randomised controlled trial of effectiveness in a standard clinical setting. Med J Aust. 2002;176:530-4.

69. Lee A, Tan S, Lim D, Winslow RM, Wong KE, Allen J, et al. Naltrexone in the treatment of male alcoholics - an effectiveness study in Singapore. Drug Alcohol Rev. 2001;20:193-9.

70. Morgenstern J, Kuerbis AN, Chen AC, Kahler CW, Bux DA, Kranzler HR. A randomized clinical trial of naltrexone and behavioral therapy for problem drinking men who have sex with men. J Consult Clin Psychol. 2012;80:863-75.

71. Morley KC, Teesson M, Reid SC, Sannibale C, Thomson C, Phung N, et al. Naltrexone versus acamprosate in the treatment of alcohol dependence: a 
multi-centre, randomized, double-blind, placebo-controlled trial. Addiction 2006;101:1451-62.

72. Morris PL, Hopwood M, Whelan G, Gardiner J, Drummond E. Naltrexone for alcohol dependence: a randomized controlled trial. Addiction. 2001;96:1565-73.

73. O'Malley SS, Jaffe AJ, Chang G, Schottenfeld RS, Meyer RE, Rounsaville B. Naltrexone and coping skills therapy for alcohol dependence. A controlled study. Arch Gen Psychiatry. 1992;49:881-7.

74. O'Malley SS, Robin RW, Levenson AL, GreyWolf I, Chance LE, Hodgkinson CA, et al. Naltrexone alone and with sertraline for the treatment of alcoho dependence in Alaska natives and non-natives residing in rural settings: a randomized controlled trial. Alcohol Clin Exp Res. 2008;32:1271-83.

75. O'Malley SS, Krishnan-Sarin S, McKee SA, Leeman RF, Cooney NL, Meandzija $B$, et al. Dose-dependent reduction of hazardous alcohol use in a placebocontrolled trial of naltrexone for smoking cessation. Int J Neuropsychopharmacol. 2008;12:589-97.

76. O'Malley SS, Corbin WR, Leeman RF, DeMartini KS, Fucito LM, Ikomi J, et al. Reduction of alcohol drinking in young adults by naltrexone: a doubleblind, placebo-controlled, randomized clinical trial of efficacy and safety. J Clin Psychiatry. 2015;76:e207-13.

77. Oslin DW, Lynch KG, Pettinati HM, Kampman KM, Gariti P, Gelfand L, et al. A placebo-controlled randomized clinical trial of naltrexone in the context of different levels of psychosocial intervention. Alcohol Clin Exp Res. 2008;32:1299-308.

78. Oslin DW, Leong SH, Lynch KG, Berrettini W, O'Brien CP, Gordon AJ, et al. Naltrexone vs placebo for the treatment of alcohol dependence: a randomized clinical trial. JAMA Psychiatry. 2015;72:430-7.

79. Petrakis IL, O'Malley S, Rounsaville B, Poling J, McHugh-Strong C, Krystal JH. Naltrexone augmentation of neuroleptic treatment in alcohol abusing patients with schizophrenia. Psychopharmacol Berl. 2004;172:291-7.

80. Petrakis IL, Poling J, Levinson C, Nich C, Carroll K, Rounsaville B. Naltrexone and disulfiram in patients with alcohol dependence and comorbid psychiatric disorders. Biol Psychiatry. 2005;57:1128-37.

81. Pettinati HM, Kampman KM, Lynch KG, Xie H, Dackis C, Rabinowitz AR, et al. A double blind, placebo-controlled trial that combines disulfiram and naltrexone for treating co-occurring cocaine and alcohol dependence. Addict Behav. 2008:33:651-67.

82. Pettinati HM, Oslin DW, Kampman KM, Dundon WD, Xie H, Gallis TL, et al. A double-blind, placebo-controlled trial combining sertraline and naltrexone for treating co-occurring depression and alcohol dependence. Am J Psychiatry. 2010;167:668-75.

83. Pettinati HM, Kampman KM, Lynch KG, Dundon WD, Mahoney EM, Wierzbicki MR, et al. A pilot trial of injectable, extended-release naltrexone for the treatment of co-occurring cocaine and alcohol dependence. Am J Addict. 2014;23:591-7.

84. Santos GM, Coffin P, Santos D, Huffaker S, Matheson T, Euren J, et al. Feasibility, acceptability, and tolerability of targeted naltrexone for nondependent methamphetamine-using and binge-drinking men who have sex with men. J Acquir Immune Defic Syndr. 2016;72:21-30.

85. Springer SA, Di Paola A, Azar MM, Barbour R, Krishnan A, Altice FL. Extended-release naltrexone reduces alcohol consumption among released prisoners with HIV disease as they transition to the community. Drug Alcohol Depend. 2017;174:158-70.

86. Tidey JW, Monti PM, Rohsenow DJ, Gwaltney CJ, Miranda R Jr, McGeary JE, et al. Moderators of naltrexone's effects on drinking, urge, and alcohol effects in non-treatment-seeking heavy drinkers in the natural environment. Alcohol Clin Exp Res. 2008;32:58-66.

87. Toneatto T, Brands B, Selby P. A randomized, double-blind, placebocontrolled trial of naltrexone in the treatment of concurrent alcohol use disorder and pathological gambling. Am J Addict. 2009;18:219-25.

88. Volpicelli JR, Alterman Al, Hayashida M, O'Brien CP. Naltrexone in the treatment of alcohol dependence. Arch Gen Psychiatry. 1992;49:876-80.

89. Volpicelli JR, Rhines KC, Rhines JS, Volpicelli LA, Alterman Al, O'Brien CP. Naltrexone and alcohol dependence. Role of subject compliance. Arch Gen Psychiatry. 1997;54:737-42.

90. Braillon A. Nalmefene in alcohol misuse: junk evaluation by the European Medicines Agency. BMJ. 2014;348:g2017.

91. Turner EH, Matthews AM, Linardatos E, Tell RA, Rosenthal R. Selective publication of antidepressant trials and its influence on apparent efficacy. N Engl J Med. 2008;358:252-60.
92. Trinquart $L$, Abbé A, Ravaud P. Impact of reporting bias in network metaanalysis of antidepressant placebo-controlled trials. PLoS One. 2012; $7:$ e35219.

93. Jansen JP, Naci H. Is network meta-analysis as valid as standard pairwise meta-analysis? It all depends on the distribution of effect modifiers. BMC Med. 2013;11:159.

94. Krahn U, Binder H, König J. A graphical tool for locating inconsistency in network meta-analyses. BMC Med Res Methodol. 2013;13:35.

95. Mills EJ, Kanters S, Thorlund K, Chaimani A, Veroniki A-A, loannidis JPA. The effects of excluding treatments from network meta-analyses: survey. BMJ. 2013:347:f5195

96. PLOS ONE Editors. Retraction: comparison of acupuncture and other drugs for chronic constipation: a network meta-analysis. Plos One. 2018;13:e0201274.

97. Bruns SB, loannidis JPA. p-curve and p-hacking in observational research. PloS One. 2016;11:e0149144.

98. Mayo-Wilson E, Li T, Fusco N, Bertizzolo L, Canner JK, Cowley T, et al. Cherrypicking by trialists and meta-analysts can drive conclusions about intervention efficacy. J Clin Epidemiol. 2017;91:95-110.

99. Noble DWA, Lagisz M, O'dea RE, Nakagawa S. Nonindependence and sensitivity analyses in ecological and evolutionary meta-analyses. Mol Ecol. 2017:26:2410-25.

100. Jackson D, Baker R, Bowden J. A sensitivity analysis framework for the treatment effect measure used in the meta-analysis of comparative binary data from randomised controlled trials. Stat Med. 2013;32:931-40.

101. Riley RD, Sutton AJ, Abrams KR, Lambert PC. Sensitivity analyses allowed more appropriate and reliable meta-analysis conclusions for multiple outcomes when missing data was present. J Clin Epidemiol. 2004;57:911-24.

102. Copas J, Shi JQ. Meta-analysis, funnel plots and sensitivity analysis. Biostat Oxf Engl. 2000;1:247-62.

103. Olkin I, Dahabreh IJ, Trikalinos TA. GOSH - a graphical display of study heterogeneity. Res Synth Methods. 2012;3:214-23.

104. Siontis KC, Hernandez-Boussard T, loannidis JPA. Overlapping meta-analyses on the same topic: survey of published studies. BMJ. 2013;347:f4501.

105. Ebrahim S, Bance S, Athale A, Malachowski C, loannidis JPA. Meta-analyses with industry involvement are massively published and report no caveats for antidepressants. J Clin Epidemiol. 2016;70:155-63.

106. Schuit E, loannidis JP. Network meta-analyses performed by contracting companies and commissioned by industry. Syst Rev. 2016;5:198.

107. Davies S. The importance of PROSPERO to the National Institute for Health Research. Syst Rev. 2012;1:5.

108. de Vrieze J. The metawars. Science. 2018;361:1184-8.

\section{Publisher's Note}

Springer Nature remains neutral with regard to jurisdictional claims in published maps and institutional affiliations.
Ready to submit your research? Choose BMC and benefit from:

- fast, convenient online submission

- thorough peer review by experienced researchers in your field

- rapid publication on acceptance

- support for research data, including large and complex data types

- gold Open Access which fosters wider collaboration and increased citations

- maximum visibility for your research: over $100 \mathrm{M}$ website views per year

At $\mathrm{BMC}$, research is always in progress.

Learn more biomedcentral.com/submission 